\title{
Referencia al artículo "Situación epidemiológica del cáncer de próstata en España”
}

\author{
Sousa Escandón A, Sabell Salgues F y Miembros del Grupo de estudio Breogan 2003-2007 \\ de la Sociedad Gallega de Urología
}

Actas Urol Esp. 2006;30(8):843

Sr. Director:

En el número de junio de Actas Urológicas Españolas se ha publicado un interesante artículo titulado “Situación Epidemiológica del Cáncer de Próstata en España" sobre el que queremos realizar algunos comentarios.

En el artículo se afirma que en España "Se han realizado estimaciones realizadas a partir de los datos disponibles, detectándose una tasa de incidencia de 45,33 por 100.000 habitantes, también entre las más bajas de Europa” y que "...es necesario ampliar el sistema de registro de cáncer para conocer la incidencia y prevalencia.".

Según los datos del 2002 del Instituto de la Salud Carlos III, el cáncer prostático es el tercer tumor que mas frecuentemente afecta a los españoles. Es cierto que los registros de cáncer urológico en España son escasos e incluso están mal realizados ya que contienen numerosos sesgos. La Sociedad Gallega de Urologia inició en 2003 un estudio denominado Breogan que pretende evaluar durante 5 años la incidencia del cáncer prostático en nuestra comunidad.

Los resultados conjuntos de los 3 primeros años han demostrado que la incidencia anual en Galicia es superior a dichas estimaciones con 143 casos por cada 100.000 varones, lo que supone una media de 1.900 nuevos casos cada año sólo en nuestra Comunidad. Obteniéndose cifras superiores a los 300 casos por 100.000 varones en el área del Hospital de Monforte, la más envejecida de Galicia, ya que el $35 \%$ de sus pacientes son mayores de 65 años.

Nuestros datos coinciden relativamente bien con los publicados para la Comunidad de Madrid por Herranz, et al. ${ }^{1}$ quienes encontraron una incidencia bruta de 100,4 casos/100.000 varones pero que alcanzaba 367,2 casos entre los varones mayores de 50 años y los 550,6 entre los mayores de 80 años. Igualmente, los estudios más recientes de la SEER que publica una incidencia en raza blanca o hispana de 147 casos por 100.000 varones $^{2}$.

Sin despreciar factores raciales, obesidad u otros; los verdaderos condicionantes de la incidencia del cáncer de próstata son la edad y el número de biopsias realizadas (cifra que a su vez depende de los criterios de uso del PSA, tanto en medicina primaria como en especializada, y de los protocolos de biopsia utilizados en cada centro).
Según las cifras publicadas por Eurostat en 2003, los varones españoles son los sextos más envejecidos de Europa y esa realidad lleva estadísticamente aparejada una incidencia elevada de cáncer prostático. Debemos plantearnos si realmente estamos infradiagnosticando esta neoplasia en nuestro país o simplemente se trata de un problema de recogida de datos. En todo caso, coincidimos con los autores en que se deben mejorar los registros de cáncer urológico en nuestro país ya que esa es la única forma de conocer realmente la entidad del problema de salud al que nos enfrentamos.

En lo relativo a la supervivencia, según los recientes datos del estudio Eurocare $2003^{3}$, España tiene tasa de pacientes vivos a los 5 años del $63 \%$, cifra que es prácticamente idéntica a la media Europea. Destacan en este aspecto países como Austria con un $82 \%$ o el grupo de Islandia, Alemania y Francia con cifras del 77, 76 y $75 \%$ respectivamente. No obstante, hemos de ser prudentes al valorar los datos de supervivencia ya que cuanto mas alto sea el porcentaje de casos diagnosticados en una población determinada (por ejemplo cuando se realizan campañas de screening), mayor será también el número de casos con valores de Gleason y PSA bajos incluidos en ese grupo y, evidentemente, estos siempre tendrán una supervivencia superior.

\section{REFERENCIAS}

1. Herranz F, Arias F, Arrizabalaga M, Calahorra FJ, Carballido J, et al. El cáncer de próstata en la comunidad de Madrid en el año 2000: Incidencia. Actas Urol Esp 2003; 27(5):323-334

2. McDavid K, Lee J, Fulton JP, Tonita J, Thompson TD. Prostate cancer incidence and mortality rates and trends in the United States and Canada. Public Health Rep. 2004; 119(2):174-186

3. Coleman M, Gatta G, Verdecchia A and the EUROCARE Working Group. EUROCARE-3 summary: cancer survival in Europe at the end of the 20th century. Annals of Oncology 2003; 14 (Suppl. 5):128-149.

Dr. A. Sousa Escandón

E-mail: alejandrosousa@hotmail.com

(Trabajo recibido el 24 de agosto 2006) 